\title{
Global characteristics of the lunar tidal modulation of the equatorial electrojet derived from CHAMP observations
}

\author{
H. Lühr ${ }^{1, *}$, T. A. Siddiqui ${ }^{2}$, and S. Maus ${ }^{3,4}$ \\ ${ }^{1}$ Helmholtz Centre Potsdam, GFZ - German Research Centre for Geosciences, Potsdam, Germany \\ ${ }^{2}$ Department of Geology and Geophysics, Indian Institute of Technology, Kharagpur, India \\ ${ }^{3}$ Cooperative Institute for Research in Environmental Science, University of Colorado, Boulder, USA \\ ${ }^{4}$ NOAA National Geophysical Data Center, Boulder, CO, USA \\ * Invited contribution by H. Lühr, recipient of the EGU Julius Bartels Medal 2011. \\ Correspondence to: H. Lühr (hluehr@gfz-potsdam.de)
}

Received: 14 October 2011 - Revised: 6 March 2012 - Accepted: 6 March 2012 - Published: 8 March 2012

\begin{abstract}
It has been known since many decades that lunar tide has an influence on the strength of the equatorial electrojet (EEJ). There has, however, never been a comprehensive study of the tidal effect on a global scale. Based on the continuous magnetic field measurements by the CHAMP satellite over 10 years it is possible to investigate the various aspects of lunar effects on the EEJ. The EEJ intensity is enhanced around times when the moon is overhead or at the antipode. This effect is particularly strong around noon, shortly after new and full moon. The lunar tide manifests itself as a semi-diurnal wave that precesses through all local times within one lunar month. The largest tidal amplitudes are observed around December solstice and smallest around June solstice. The tidal wave crest lags behind the moon phase. During December this amounts to about 4 days while it is around 2 days during other times of the year. We have not found significant longitudinal variations of the lunar influence on the EEJ. When comparing the average EEJ amplitude at high solar activity with that during periods of solar minimum conditions a solar cycle dependence can be found, but the ratio between tidal amplitude and EEJ intensity stays the same. Actually, tidal signatures standout clearer during times of low solar activity. We suggest that the tidal variations are caused by a current system added to the EEJ rather than by modulating the EEJ. Gravitational forcing of the lower atmosphere by the moon and the sun is assumed to be the driver of an upward propagating tidal wave. The larger tidal amplitudes around December solstice can be related to stratospheric warming events which seem to improve the conditions for upward propagation.
\end{abstract}

The results described here have to large extent been presented as a Julius-Bartels Medal Lecture during the General Assembly 2011 of the European Geosciences Union.

Keywords. Ionosphere (Electric fields and currents; Equatorial ionosphere) - Meteorology and atmospheric dynamics (Waves and tides)

\section{Introduction}

The term equatorial electrojet (EEJ) stands for an intense electric current flowing along the dip-equator in the ionospheric E-layer. The intensity of the EEJ is influenced by many drivers. These include thermospheric winds (e.g. Maus et al., 2007), interplanetary electric field (e.g. Manoj et al., 2008), solar extreme ultra-violet (EUV) flux (e.g. Alken and Maus, 2007), and non-migrating solar tides (e.g. England et al., 2006; Lühr et al., 2008, 2011).

It has long been recognised that lunar tides also have an influence on the EEJ intensity. An early comprehensive study was presented by Bartels and Johnston (1940). From their observations at the Huancayo observatory they deduced some important features of the lunar influence on the electrojet. For the months around December solstice during solar maximum years they determined that the largest EEJ amplitudes were around $110 \mathrm{nT}$, and the semi-monthly wave (lunar tide) had amplitudes of more then $20 \mathrm{nT}$. Conversely, around June solstice the electrojet was weakest $(\sim 70 \mathrm{nT})$ and the lunar tide reached some $12 \mathrm{nT}$. The phase of the tidal wave 
lagged behind the lunar phase by $2-3 \mathrm{~h}$. The proper interpretation of these single-station observations was quite demanding because all large-scale variations, including the diurnal variation, are superimposed on the EEJ signal of interest. More recent studies have used nearby low-latitude observatories for removing the large-scale background fields. This topic of lunar influence on the electrojet has been revisited later by a number of other researchers (e.g. Onwumechili, 1963; Rastogi and Trivedi, 1970). All of these later studies confirm by and large the findings of Bartels and Johnston (1940). For a comprehensive overview of past studies see e.g. Onwumechili (1997) and references therein.

A serious limitation of EEJ studies from observatories is that they record variations generally at a single longitude. It is therefore difficult to get a global overview of the EEJ from ground stations. Magnetic field satellite missions like Ørsted and CHAMP can help in this respect and map out the EEJ distribution world wide (e.g. Jadhav et al., 2002; Lühr et al., 2004). Only after considering a sufficient amount of satellite data the true longitudinal variation of the EEJ has been determined for the first time by Alken and Maus (2007). They presented an empirical model of the EEJ intensity. Based on that model it was possible to reveal details of the non-migrating solar tidal signals in the EEJ (e.g. Lühr et al., 2008, 2011).

So far there is no comprehensive global study of the lunar influence on the EEJ. Several questions are awaiting answers. Is there a longitudinal dependence of the tidal wave? What is the solar flux dependence of the lunar tide? Is there a difference in the signal between new moon and full moon phases? Recently, indications have been presented that meteorological phenomena can modify the EEJ. For considering these open issues we have made use of 10 years of CHAMP magnetic field measurements. The time period ranging from 2000 to 2010 covers the solar maximum in the first part and the deep and extended recent minimum in the second.

In the sections to follow we will first introduce the data and the approach of deriving the lunar tidal signal. Then we present the derived results. In the discussion section we interpret the findings and compare them with previous publications. Finally conclusions will be drawn.

\section{Dataset and analysis approach}

The CHAMP satellite was launched on 15 July 2000 into a circular, near-polar (inclination: $87.2^{\circ}$ ) orbit at $456 \mathrm{~km}$ altitude (Reigber et al., 2002). Over its 10-year life time the orbit decayed by about $50 \mathrm{~m}$ per day until its re-entry into the atmosphere on 19 September 2010. The orbital plane precessed through local time at a rate of $1 \mathrm{~h}$ per 11 days. CHAMP needed 131 days to sample all local times, when considering both ascending and descending crossings of the equator. Within a time period of 5 years there is just an even distribution of local time sampling of all seasons. These two periods, 131 days and 5 years, are thus important to consider if one wants to avoid a seasonal/local time beating.

The electrojet current strength was deduced from magnetic field measurements onboard CHAMP. Pre-processed vector field data are available at $1 \mathrm{~Hz}$ rate. For maintaining the accuracy over the full mission life time these data are calibrated routinely against an absolute scalar magnetometer. As a first step of data evaluation all the magnetic field contributions from sources other than ionospheric currents have been subtracted. These are the geomagnetic main field, crustal field and magnetospheric contributions. For this purpose the POMME model (Maus et al., 2006) was used to represent the various source terms. In addition the contributions from large-scale ionospheric currents, here mainly the Sq current, have to be removed. The latter part is determined by an orbit-by-orbit fitting procedure. Finally the electrojet magnetic signature remains. This is used in an inversion process for deriving latitudinal profiles of the EEJ current density. A more detailed description of the EEJ determination can be found in Lühr et al. (2004). For the study presented here we consider the EEJ peak current density at the time of equator crossing. This gives just one number per pass that characterises the intensity of the electrojet.

CHAMP circled the Earth about 15.5 times per day. This adds up to more than 28000 crossings of the dayside equator within 5 years. All these data are sorted by moon phase and solar local time. For calculating the new moon epoch, $\mathrm{MJD}_{\mathrm{NM}}$, we used the formula

$\operatorname{MJD}_{\mathrm{NM}}=5.5970+29.53058886 N-\left(133 \times 10^{-12}\right) N^{2}$

MJD is the modified Julian day after 1 January 2000 , 00:00 UT and $N$ is the sequence number of new moons in this millennium. It is known that the time between subsequent new moon epochs varies due to the eccentricity of the moon and Earth orbits. But this oscillatory deviation amounts only to a few hours and can be neglected in our statistical study. The time between new moons is divided evenly into 24 parts. They are termed "moon phase (MP)". All new moons occur at the phase $0 \mathrm{~h}$ MP and all full moons at $12 \mathrm{~h}$ MP.

The EEJ current readings have been sorted into bins of $2 \mathrm{~h}$ in moon phase and $1 \mathrm{~h}$ in local time (LT). The local time sector considered here lasts from 06:00 to 18:00 LT. Outside of that sector the EEJ signal is weak. Such a moon phase versus local time gridding creates 144 bins. When stacking all moon phases and considering the readings from the first 5 years of the mission we get on average $\sim 200$ samples per bin. This is a convenient sample number for deriving statistically significant results. For representing the bin averages we are taking the median rather than the mean in order to reduce the influence of disturbed days. Over the course of the study we will further subdivide the samples for investigating seasonal and longitudinal dependencies. 


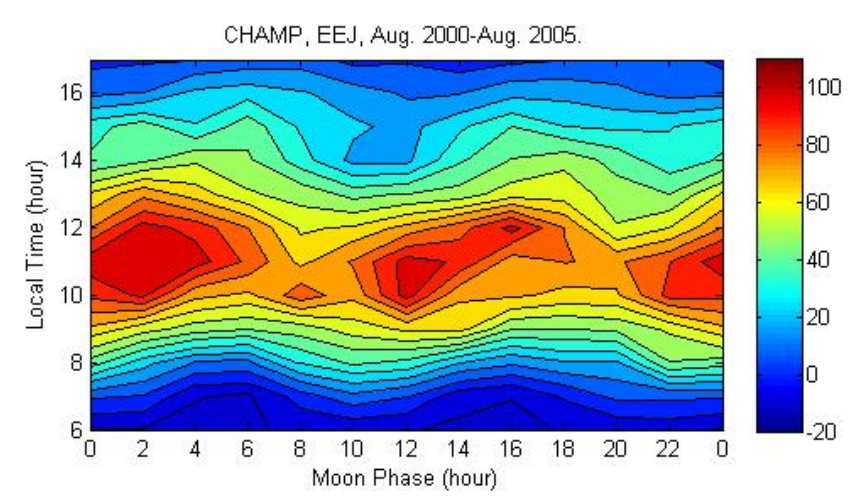

Fig. 1. Local time variation of the electrojet lunar tidal signal. Global averages of EEJ peak current densities $\left(\mathrm{mA} \mathrm{m}^{-1}\right)$ are shown.

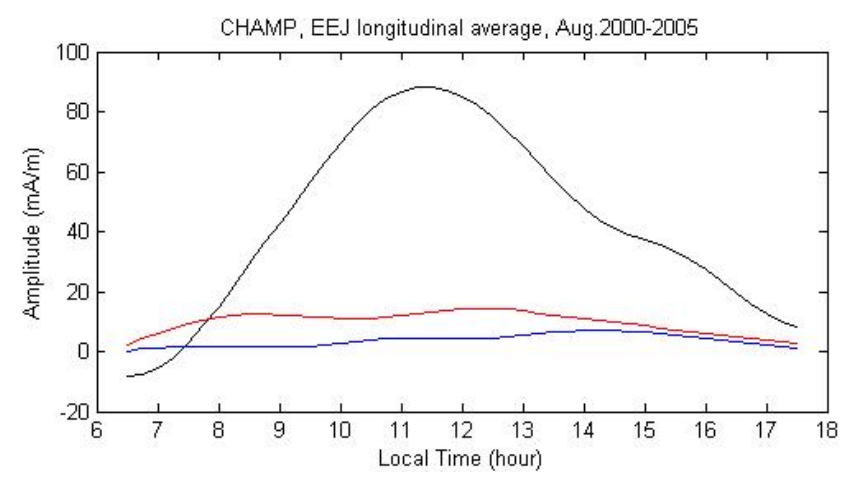

Fig. 2. Local time variation of the amplitudes from some tidal harmonics over a lunar month: (black) constant part, (blue) first harmonic, (red) second harmonic.

\section{The average EEJ lunar signal}

For our study we have divided the full CHAMP data set into two parts covering 5 years each. The first interval, August 2000-2005, coincides with a period of enhanced solar activity and the second, August 2005-2010, reflects solar minimum conditions. Since EEJ features are known to be more prominent during solar maximum years, we will focus our detailed studies on the first interval and consider the second interval later.

Figure 1 shows the local time versus moon phase distribution of the EEJ intensity. Most prominent is the well-known local time variation of the EEJ current density reaching peak values around 11:00 LT. Secondly, the intensity is modulated by the semi-diurnal lunar tide. Highest current densities are found shortly after the moon phases $0 \mathrm{~h}$ (new moon) and $12 \mathrm{~h}$ (full moon). In order to investigate the lunar signal more quantitatively we have performed harmonic analyses over a full lunar month separately for each local time hour. In Fig. 2 the local time dependence of some harmonic signals are shown. The constant part (black line), which is independent of lunar phase, reflects the typical diurnal variation

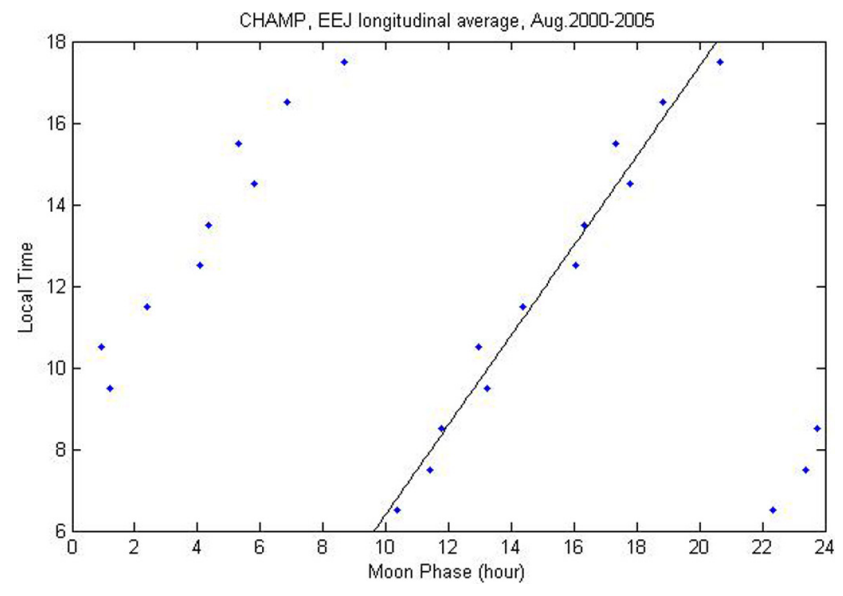

Fig. 3. Phase propagation of the semi-monthly lunar tide as a function of moon phase. Dots mark for each local time hour the moon phase when the tidal wave crest is observed.

of the EEJ intensity reaching peak current densities of some $90 \mathrm{~mA} \mathrm{~m}^{-1}$ around 11:00 LT. Of particular interest for this study is the semi-monthly oscillation (red curve). It attains amplitudes up to $15 \mathrm{~mA} \mathrm{~m}^{-1}$ around noon. Although small (less than $8 \mathrm{~mA} \mathrm{~m}^{-1}$ ) there is also a monthly oscillation. Its phase indicates a slight dominance of the new moon tidal amplitude over that of the tide at full moon.

We inspected also the phase variation of the second harmonic signal. Figure 3 shows for each local time hour at which moon phases the peaks of the semi-diurnal wave appeared. We obtain a consistent pattern of phase propagation with aging moon. A regression line has been fitted to the phase values that were determined independently for each local time hour. The formula for the linear fit, which is obtained with a high correlation coefficient $(0.98)$, can be used to estimate the local time (LT) for all moon phases (MP)

$\mathrm{LT}=1.2(\mathrm{MP}-12 \mathrm{~h})+8.5 \mathrm{~h}$

For convenience of application we have centred the equation at full moon. There are two things to note, (1) the scale factor is somewhat larger than the expected factor 1, (2) the local time of the wave crest lags behind the moon phase. Due to the somewhat steeper phase curve the lag time is $4 \mathrm{~h}$ in the morning, $3 \mathrm{~h}$ around noon and about $2 \mathrm{~h}$ in the evening. This topic will be discussed in more details in Sect. 4.1.

\subsection{Seasonal dependence of lunar tide}

The results presented above reflect average conditions for the 5 years (August 2000-2005) considered. It had been mentioned earlier that the EEJ tidal signal varies from season to season (e.g. Bartels, 1936). Due to the large amount of readings available we can afford to subdivide the samples into seasons. Here the Lloyd's seasons have been used; December solstice is represented by the months November to February, 

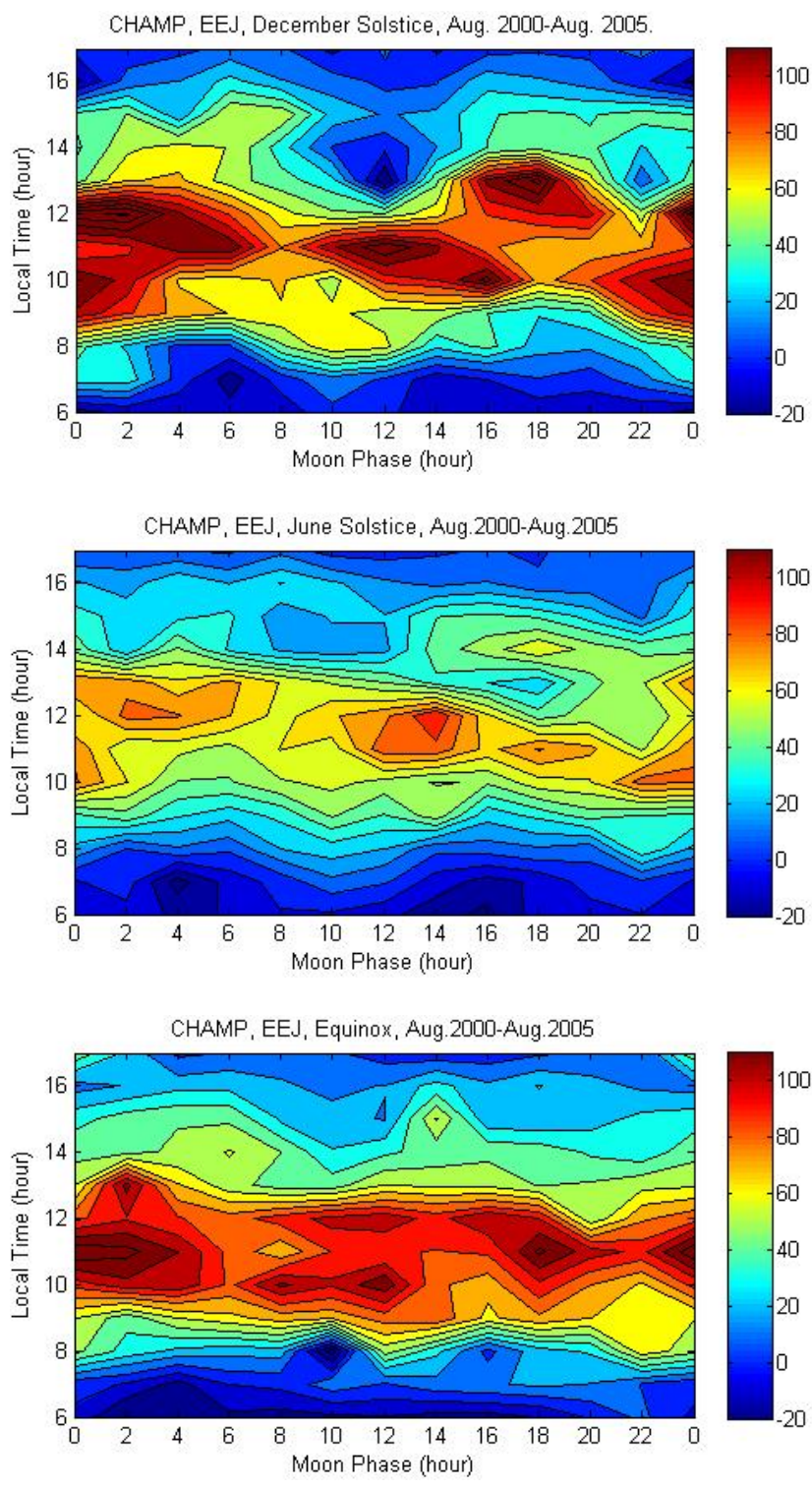

Fig. 4. The same as Fig. 2, but separately for the seasons around December solstice, June solstice and equinoxes.

June solstice by May to August and the combined equinoxes by the months March, April, September, October.

Figure 4 presents the EEJ current density distributions in local time versus moon phase frames separately for the three seasons. There are indeed significant differences between the tidal signatures. The EEJ amplitude modulation by the lunar tides is best reflected during December season. High current densities are well aligned with phase fronts of the lunar tide. During the months around June solstice the EEJ strength is overall, as expected, much weaker. Still there is a clear indication of a semi-diurnal oscillation shifting in phases over half a lunar month. For the equinox months we obtain a more complex distribution of EEJ intensities. Around new moon highest current densities appear, but at full moon the situation is quite different. Here we find patches of strong electrojet current next to weaker areas in the middle of the day.

Some characteristic quantities of the EEJ tidal features are summarised in Table 1 separately for the three seasons. In the second column the noon-time EEJ peak current density averaged over all lunar phases is listed for comparison. The semi-monthly variation is regarded as the prime lunar tide. The amplitudes listed are averages over the local time sector 10:00-14:00 LT. Phase delays presented reflect the times in moon phase by which the tidal bulges lag behind. With the help of a linear regression we find the slope by which the phase front of the lunar tide advances in local time for every hour of moon phase. The table by and large confirms the visual impression from Fig. 4. Lunar tides are best developed around December. Here we find by far the largest tidal amplitudes. Even though the EEJ is strongest during that season, the modulation ratio of about $25 \%$ is the highest. Also the linear fit to the phase propagation in local time of the tidal wave is best during that season. Around the middle of the year the EEJ is weakest. But even weaker is the tidal wave, resulting in a modulation ratio of only $14 \%$. Consequently, the linear fit to the tidal wave propagation is slightly worse than that of the months around December. During equinoxes the EEJ is strong again, but the tidal modulation ratio of $16 \%$ is comparable with that of June. In general, the tidal signal is not so clear during equinox, which is also reflected by the much reduced correlation coefficient. Opposed to the other two seasons the data distribution for the equinox months is rather uneven. Some of the bins in the local time versus moon phase frames (Figs. 4 and 8) stayed even empty and had to be filled by interpolation.

Concerning the behaviour of the tidal wave, we find only for December solstice a slope for the phase propagation larger than 1. This is consistent with the faster change in local time as mentioned above for the annual average. For the other two seasons we obtain the expected slope 1 . The phase delay varies from season to season, with largest values around December and smallest during equinoxes.

\subsection{Longitudinal dependence of the lunar tide}

The magnetic equator is located over certain longitude ranges in the Northern Hemisphere and over other longitudes in the Southern Hemisphere. This latitudinal displacement of the EEJ may cause some longitude dependences of the tidal signal. In order to check this we have further subdivided the seasonal samples into $120^{\circ}$ wide longitude sectors. By doing so the number of entries per bin in the local time versus moon phase frames has become marginally small. On average there are 20 reading per bin. In reality however, there are some bins that stay even empty in particular for equinox months. In such cases we have closed the gaps by interpolation.

Figure 5 presents a comparison between tidal signals from two longitude sectors. Here again individual plots for all three seasons are shown. The longitude sectors chosen are 
Table 1. Characteristic features of the EEJ lunar tides separately for the three Lloyd's seasons. The phase propagation of the tidal wave has been determined by linear regression.

\begin{tabular}{lccccc}
\hline Season & Noon EEJ amplitude & Amplitude lunar tide & Phase delay & Slope of tide & Correlation coefficient \\
\hline Dec solstice & $96 \mathrm{~mA} \mathrm{~m}^{-1}$ & $23.5 \mathrm{~mA} \mathrm{~m}^{-1}$ & $3.8 \mathrm{~h} \mathrm{MP}$ & 1.11 & 0.97 \\
Jun solstice & $69 \mathrm{~mA} \mathrm{~m}^{-1}$ & $9.2 \mathrm{~mA} \mathrm{~m}^{-1}$ & $2.3 \mathrm{~h} \mathrm{MP}$ & 1.01 & 0.92 \\
Equinoxes & $94 \mathrm{~mA} \mathrm{~m}^{-1}$ & $15.5 \mathrm{~mA} \mathrm{~m}^{-1}$ & $1.7 \mathrm{~h} \mathrm{MP}$ & 1.02 & 0.83 \\
\hline
\end{tabular}
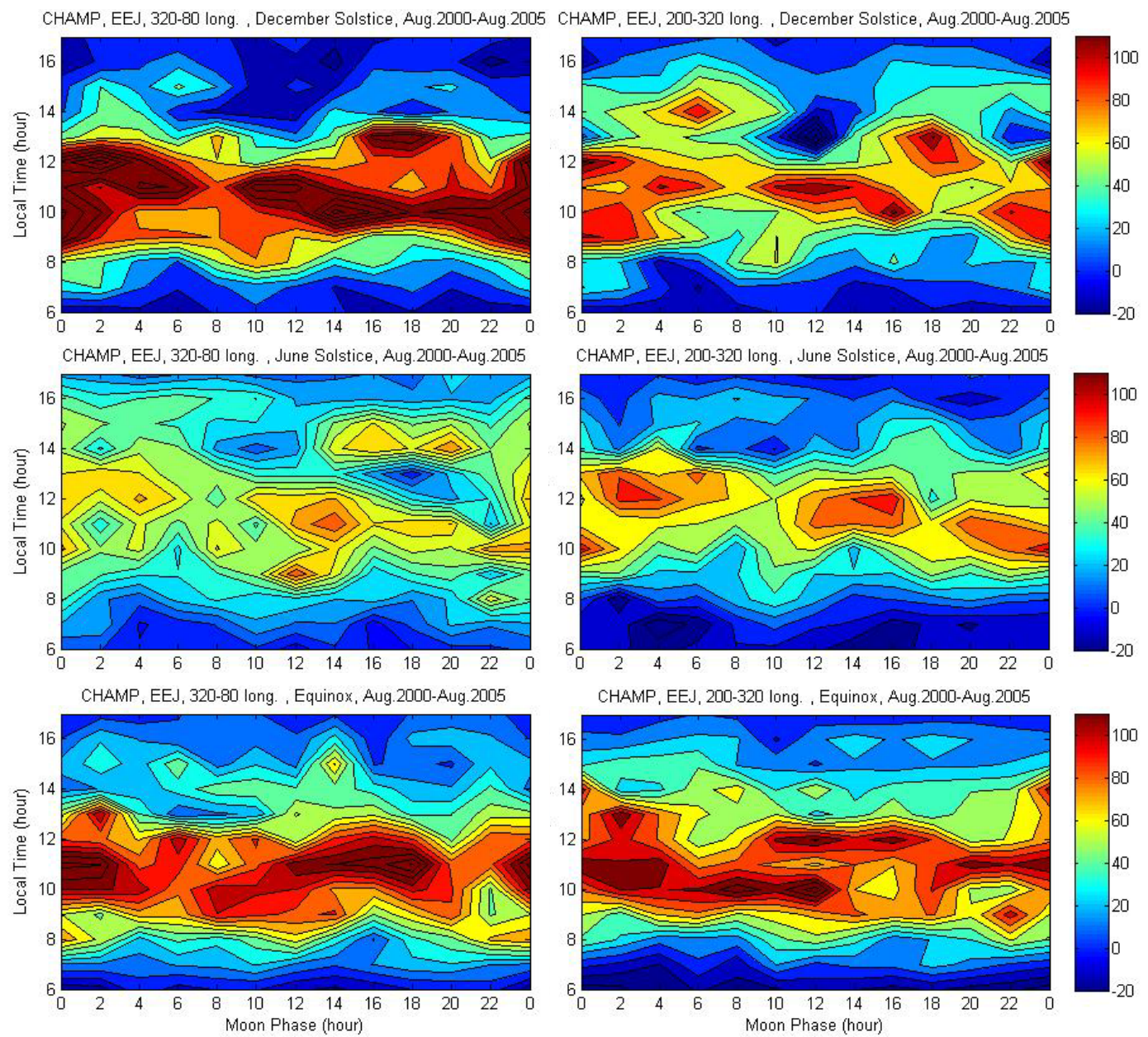

Fig. 5. The same as Fig. 4, but separately for the sectors $320^{\circ}-80^{\circ}$ (left) and $200^{\circ}-320^{\circ}$ of longitudes (right).

$320^{\circ}-80^{\circ} \mathrm{E}$ and $200^{\circ}-320^{\circ} \mathrm{E}$. They represent a good contrast in latitudinal displacement of the EEJ. The average latitude of the dip-equator over the longitude range $320^{\circ}-80^{\circ} \mathrm{E}$ is $8.8^{\circ} \mathrm{N}$ and for $200^{\circ}-320^{\circ} \mathrm{E}$ it is $6.4^{\circ} \mathrm{S}$.

When comparing the seasonal plots from the two latitude sectors there are no obvious differences emerging with respect to the tidal features. Many tidal details repeat in both sectors. The average strength of the EEJ, however, differs from sector to sector. It is interesting to note that the EEJ intensity around December dominates in the sector $320^{\circ}-80^{\circ} \mathrm{E}$ of longitude although the dip-equator here has a significant northward bias. Conversely, the EEJ around June is stronger in the $200^{\circ}-320^{\circ} \mathrm{E}$ longitude sector with its average latitude of $6.4^{\circ} \mathrm{S}$. This seasonal/longitudinal dependence of the EEJ is primarily caused by non-migrating solar tides (see Lühr et al., 2008, 2011). The longitude dependence of the EEJ intensity, however, is not at the focus of this study. We are not presenting numerical values for the tidal waves from the different longitude sectors because the significance is questionable due to the small sample numbers. In spite of that limitation it may be concluded that the lunar influence on the EEJ is not much dependent on longitude. 


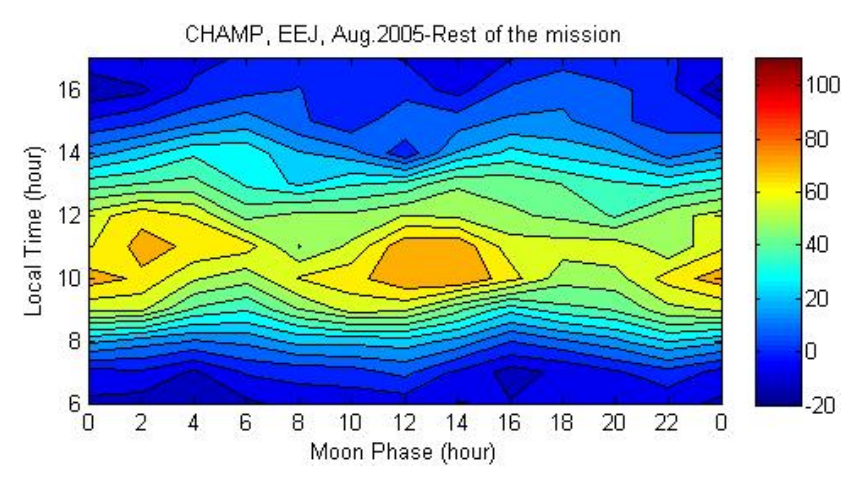

Fig. 6. The same as Fig. 1, but for the solar minimum years August 2005-2010.

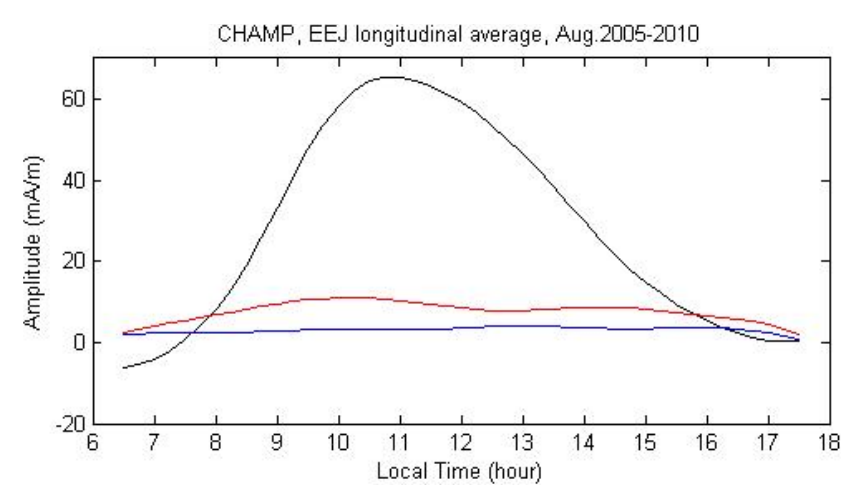

Fig. 7. The same as Fig. 2, but for the solar minimum years August 2005-2010.

\subsection{Solar cycle dependence of the lunar tide}

So far we have presented EEJ observations from the first 5 years of the CHAMP mission. During this period, August 2000-2005, the solar activity was quite high. The average EUV solar flux index amounted to F10.7 $=145 \pm 44 \mathrm{sfu}$. For comparison the remaining 5 years of the CHAMP mission, August 2005-2010, were also considered. This coincides with a period of very low solar activity. We obtain an average solar flux index of F10.7 $=76 \pm 16$ sfu. Accordingly, weaker EEJ currents are expected.

At first we look again at the global average of the EEJ features during solar minimum years. Figure 6 shows equivalent to Fig. 1 diurnal variations of the EEJ intensity over a full lunar month. Tidal features are quite similar, but current densities are smaller, as expected. In order to get more quantitative results we have plotted in Fig. 7 the diurnal variations of some tidal harmonics equivalent to Fig. 2. The mean EEJ current density peaks at $65 \mathrm{~mA} \mathrm{~m}^{-1}$ around 11:00 LT. For the prime lunar tide (semi-diurnal) we derive an amplitude of about $10 \mathrm{mAm}^{-1}$. The monthly wave stays below $4 \mathrm{~mA} \mathrm{~m}^{-1}$. When compared to the high solar activity period the average modulation of the EEJ intensity by the lunar tide amounts to about $16 \%$ in both cases. The propagation of the
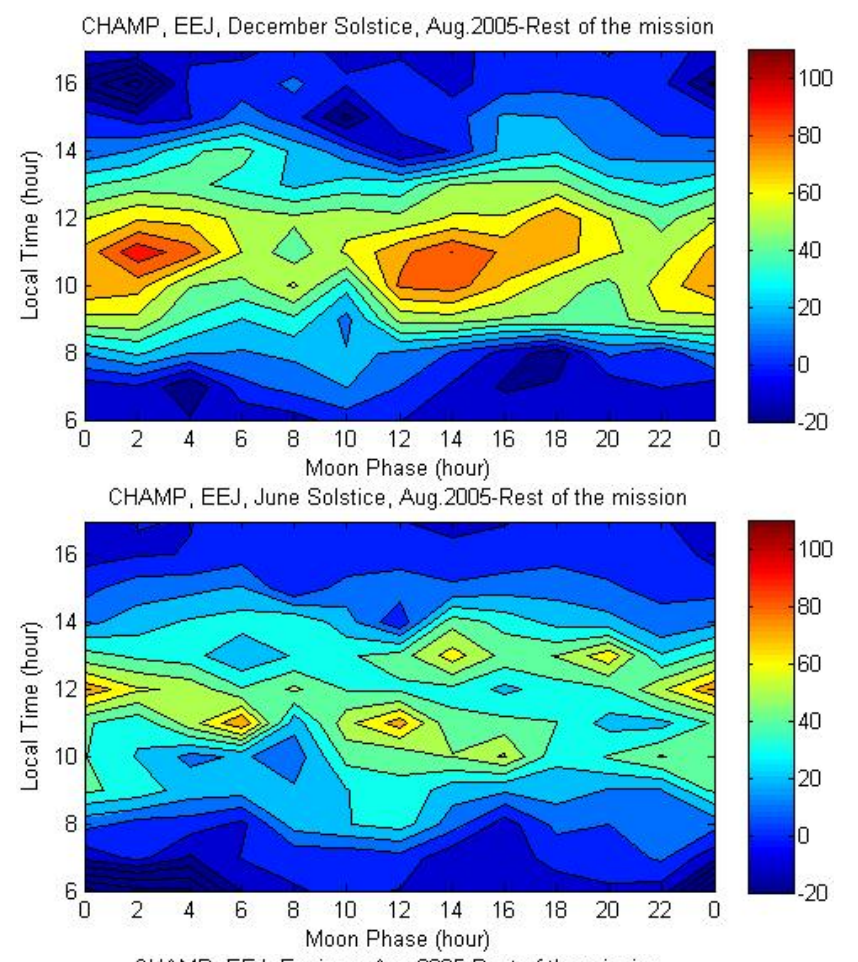

CHAMP, EEJ, Equinox, Aug.2005-Rest of the mission

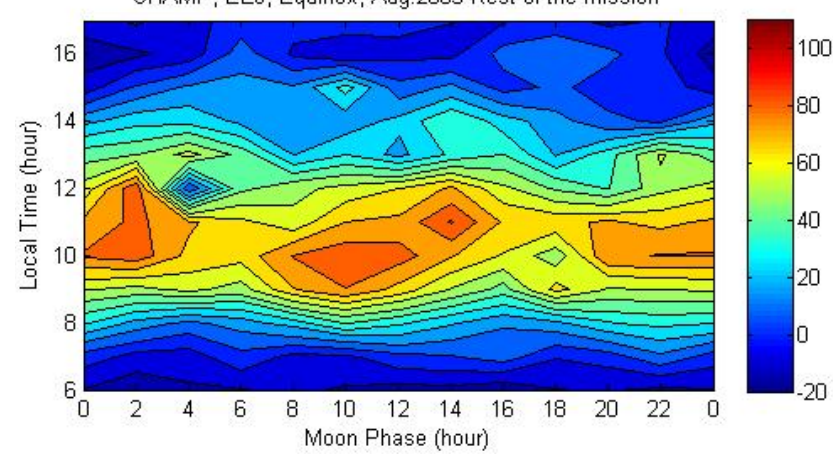

Fig. 8. The same as Fig. 4, but for the solar minimum years August 2005-2010.

tidal wave is also as expected. We can again express it by a linear relation

$\mathrm{LT}=1.03(\mathrm{MP}-12 \mathrm{~h})+8.9 \mathrm{~h}$

In this case the slope is closer to the expected 1 and the correlation coefficient of the linear regression is even higher (0.99). Over all, we may state that the tidal features of the EEJ have not changed much with solar activity.

Here it may be of interest again to look into the seasonal behaviour during solar minimum years. In the same way as Fig. 4 the lunar tidal structures are shown separately for each season in Fig. 8. It is immediately evident that the tidal signals show up clearer during solar minimum years than during active years. This is probably due to the much smaller number of disturbing magnetic storms. Main features of the tides 
Table 2. The same as Table 1, but for the period August 2005-2010.

\begin{tabular}{lccccc}
\hline Season & Noon EEJ amplitude & Amplitude lunar tide & Phase delay & Slope of tide & Correlation coefficient \\
\hline Dec solstice & $72 \mathrm{~mA} \mathrm{~m}^{-1}$ & $13 \mathrm{~mA} \mathrm{~m}^{-1}$ & $4.2 \mathrm{~h} \mathrm{MP}$ & 1.09 & 0.98 \\
Jun solstice & $47 \mathrm{~mA} \mathrm{~m}^{-1}$ & $7 \mathrm{~mA} \mathrm{~m}^{-1}$ & $0.9 \mathrm{~h} \mathrm{MP}$ & 0.97 & 0.92 \\
Equinoxes & $75 \mathrm{~mA} \mathrm{~m}^{-1}$ & $10 \mathrm{~mA} \mathrm{~m}^{-1}$ & $2.3 \mathrm{~h} \mathrm{MP}$ & 0.90 & 0.93 \\
\hline
\end{tabular}

are again listed in Table 2. The entries can directly be compared with those in Table 1 . As expected, the intensity of the noon-time EEJ is reduced during the minimum years. More important for this study is the ratio of modulation by the lunar tide. We obtain $18 \%, 15 \%$ and $13 \%$ for December, June solstice and equinoxes, respectively. When comparing to the results from the first half of the CHAMP mission we see that the relative strength of the tide has become weaker during the months around December, but for the other seasons we find about the same percentages.

Concerning the phase propagation of the semi-monthly lunar wave we find here similar lag times for the three seasons as given in Table 1. Long delays of more than $4 \mathrm{~h}$ in moon phase occur around December solstice and times of $1-2 \mathrm{~h}$ are found for the other two seasons. As mentioned earlier, the slope of phase propagation is again larger than 1 during months around December and close to the expected factor 1 for the rest of the year. The correlation coefficients for the linear regressions are consistently higher than the ones listed in Table 1. This again is an indication for the clearer tidal signals during low solar activity years.

\section{Discussion}

The long and homogeneous CHAMP dataset allows for a comprehensive investigation of the influence of lunar tides on the equatorial electrojet. Never before have the related phenomena been studied on global scale in such detail. The 10 years of observations are favourable in many respects; (1) within 5 years CHAMP samples all local times evenly in every season, and we analysed two of these 5-year periods; (2) the solar and geomagnetic activity conditions were very different during the two considered periods; (3) CHAMP crossed the equator more than 56500 times on the dayside, providing a large sample number suitable for studying the statistical properties of many tidal parameters. On the other hand, single-satellite observations are not suitable to study the day-to-day variability of the EEJ.

\subsection{Characteristics of the lunar influence on the electrojet}

It has long been recognized that the EEJ intensity is modulated by the moon phase. Enhancements of current density appear at times and locations where the moon is over-

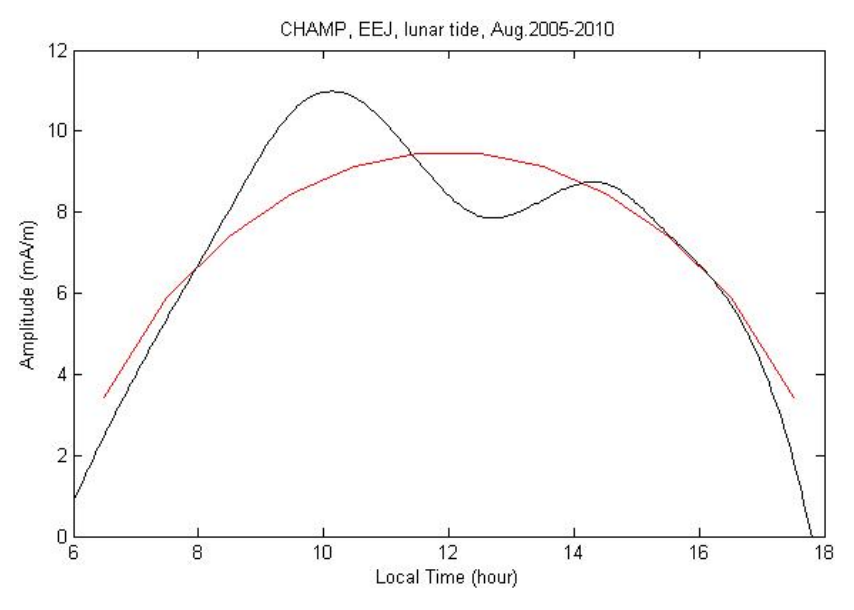

Fig. 9. Diurnal variation of lunar tidal amplitude (black curve) compared with the square root of the cosine of the solar zenith angle (red curve). The latter function reflects the electron density variation of a Chapman layer.

head or at the antipodes. In this sense the EEJ lunar signal behaves similar to the tidal bulge on the oceans. The mechanism, however, that enhances the EEJ is quite different. The gravitational attraction of moon and sun modifies slightly the pressure and temperature of the low atmosphere. These tiny effects at Earth surface propagate upward as tidal wave with increasing amplitude (e.g. Chapman and Lindzen, 1970). The lunar semi-diurnal wave seems to drive a current system in the E-layer at dip-latitudes that adds to the EEJ. When analysing the amplitude of the semi-monthly lunar wave we find an almost constant current density of this second harmonics over many hours of local time (see Figs. 2 and 6). Conversely, the EEJ intensity itself varies strongly over the same local time sector and reverses even sign in the morning. The diurnal variation of the semi-diurnal tidal current density can be approximated reasonably well by the square root of the cosine of the solar zenith angle (see Fig. 9). This cosine function reflects the electron density variation of a Chapman layer. We suggest therefore that the amplitude of the EEJ lunitidal component varies as the E-region conductivity. In order to verify this suggestion it is recommended to check, for example, wind measurements in the mesosphere, lower thermosphere (MLT) region, and see what kind of lunar signal they show at low latitudes. Such kind of data should be available from UARS or TIMED satellites. 
For most of the months (equinoxes and June solstice) the local time of the semi-diurnal tidal wave crest follows the motion of the moon, but the crest appears delayed by $1-2 \mathrm{~h}$ in moon phase. This phase behaviour may be compared with the lunitidal signature in surface air pressure. For the station Batavia Bartels and Kertz (1952) presented in their Fig. 3 a delay of about $0.5 \mathrm{~h}$ in moon phase for the seasons equinox and June solstice. Different from that they find delays around $1.75 \mathrm{~h}$ of MP during the months December, January, February. This is consistent with our larger phase delay of the lunitidal wave in the EEJ for these months. An interesting finding is the steeper slope of the phase line that we observe only during December solstice months. The delay is largest right after half-moon epochs when the crest is expected in the morning. The delay becomes progressively shorter over the course of the moon phase until the crest approaches the evening. As a consequence, the EEJ shows no lunar tide in the morning during the moon phases $08.5-09.5 \mathrm{~h}$ and $20.5-$ $21.5 \mathrm{~h}$ of MP. It looks as if at E-layer heights the tidal wave disappears around half-moon, the time when gravitational forcing is smallest, and builds up again after 1-2 days with a large phase delay that reduces when the moon phase approaches full or new moon. We have no immediate explanations for this special behaviour during the months around December. It might be informative to process the ground air pressure variations in the same way as we did it with the EEJ data and check the phase behaviour.

Our studies reveal that the features of the lunar tide do not vary much with longitude. However, the amplitude of the semi-monthly oscillation varies in the same way as the longitude dependence of the EEJ intensity, and it also tracks the seasonal variation. This is consistent with the idea of an upward propagating tidal wave driving a current along the dip-equator, which has an intensity proportional to the local ionospheric conductivity. Based on the finding of little longitudinal dependence we may conclude that EEJ lunar variations can be studied well by any equatorial observatory.

\subsection{Dependence on solar activity}

The current density driven by the tide at the dip-equator is not too strong. On annual average the semi-monthly amplitude amounts to $15 \mathrm{~mA} \mathrm{~m}^{-1}$ during the active solar period and $10 \mathrm{~mA} \mathrm{~m}^{-1}$ at solar minimum. The average solar flux indices, F10.7, during these periods have been 145 and $76 \mathrm{sfu}$, respectively. Earlier radar studies have revealed that the height-integrated ionospheric conductivity varies as the square root of the solar flux index (e.g. Robinson and Vondrak, 1984; Schlegel, 1988). When considering this dependence we obtain for the conductivity ratio 1.4 and for the current density ratio 1.5 . This again can be seen as a confirmation for the strong dependence of the lunar signature on the ionospheric conductivity.

When comparing the amplitudes of lunar tides between the two analysed periods we find particularly strong tidal effects around December. This enhancement cannot be explained by higher solar flux. There seem to be other processes influencing the strength of the EEJ lunar tide. We will revisit this subject in the next section.

The tidal phase behaviour is very much the same at high and low solar activity periods. Longest delays of more than $4 \mathrm{~h}$ of moon phase are found consistently around December solstice months. Overall, the tidal signal shows up clearer during times of low solar activity. Obviously, the upward propagation of the tidal wave to the ionosphere is less perturbed during solar quiet periods. Also the times without tidal signal around half-moon phases during December solstice months is present at both activity states.

\subsection{Comparison with other tides}

The features of the lunar tides differ very much from the solar tidal modulation of the EEJ. Only recently the effect of nonmigrating tides on the EEJ has been studied in more details (e.g. Lühr et al., 2008, 2011). For example the diurnal eastward propagating tide with wavenumber 3 (in short DE3) has a large impact on the EEJ longitudinal distribution. DE3 amplitudes maximise around August and practically disappear around December. Lunar tides behave just oppositely. They are largest around December and smallest around July. Reasons for the differences are the very different driving mechanisms. In case of the lunar tide it is a gravitational excitation of a semi-diurnal tidal wave and in case of DE3 it is an interaction of the migrating diurnal tide with the strong release of latent heat in the tropical troposphere (Hagan and Forbes, 2002). This interaction causes among others a longitudinal pattern of alternating zonal winds that modulate the EEJ intensity (for more details see Oberheide et al., 2009; Lühr et al., 2011, and references therein). The DE3 non-migrating tide has a significantly larger impact on the EEJ. Modulation ratios go up to $50 \%$ in August (Lühr et al., 2011). In case of the lunar tide the ratio amounts typically to $15 \%$. Only around December during the active years we found ratios up to $25 \%$.

The non-migrating solar tides cause distinctive longitudinal patterns of EEJ intensity, which vary with seasons. Therefore global observations of the EEJ are required to identify the features of these tidal contributions. This is the reason why only recently, based on satellite observations, their tidal features have been discovered. Measurements from singe observatories are not sufficient for monitoring non-migrating tides. This is not valid for lunar tides.

We showed that the EEJ lunar signal is largest around December. Already Bartels and Johnston (1940) noted this fact when analysing EEJ observations from Huancayo. They even mentioned the occurrence of so-called "big L days" referring to large lunar tides. Interestingly, all their "big L days" occurred around January. Only recently it was found that sudden polar stratospheric warming (SSW) events have an influence on the lunitidal signatures in the ionosphere at 
equatorial latitudes (e.g. Chau et al., 2009). Based on data from Jicamarca (radar and magnetometer) Fejer et al. (2010) reported that the lunar modulation of the vertical plasma drift and EEJ intensity increased significantly for a few weeks after the onset of an SSW. It is well known that SSWs occur predominately in the Northern Hemisphere and commonly start several weeks after December solstice. Park et al. (2012) have correlated the stratospheric temperature difference between North Pole and $60^{\circ}$ latitude with the amplitude of the EEJ modulation by the lunar tide. They find a convincing one-to-one correspondence between SSW events and large lunitidal signals in the EEJ. We thus may conclude that the on average larger EEJ lunar tides around December are caused by the influence of SSWs. In this context it is interesting to note that Bartels and Kertz (1952) reported for the lunitidal amplitude of ground-level air pressure largest values around June solstice and at least $20 \%$ smaller ones around December solstice. This may be seen as an indication that the propagation conditions from the lower atmosphere to the ionosphere are season dependent and that SSW events are especially efficient in improving the upward propagation of the lunitidal wave.

Besides the semi-monthly activity variation we observe also a lunar monthly wave. The effect of this latter wave is that tidal amplitudes are somewhat smaller around full moon than around new moon. This is a puzzling result since the gravitational forces are the same for both moon phases. There must be some other effects influencing the ionospheric tidal signal. When looking for example at the June tidal signals in Figs. 4 and 8 we see a gradual shift of the noon-time maximum from about 13:00 LT at new moon epoch to earlier local times over the course of a lunar month. Around new moon the EEJ peak intensity jumps back again from 10:00 LT to about 13:00 LT. This tilted activity front seems to be repeated approximately $3 \mathrm{~h}$ later. Similarly tilted activity patterns can also be identified in the December frames of Figs. 4 and 5. There seems to be a modulation of the EEJ intensity by a quasi 3-h period wave which is also synchronised with the lunar phase. Such a short-period wave has never been mentioned before in literature, and it causes a difference between the tidal amplitudes at full and new moon. The 3-h wave affects the tidal amplitudes at the two moon phases just in opposite directions. This is the reason for the apparent monthly lunar wave.

We had a closer look at the samples in the individual bins and found that the tilted activity fronts are caused by a beating between the local time variation of the CHAMP samples and the moon phase. During one lunar cycle the CHAMP orbit precesses towards earlier local times by almost $3 \mathrm{~h}$. The activity fronts in Figs. 4 and 8 follow exactly the local time precession. Thus these features are artefacts caused by the satellite sampling. This statement can be validated by repeating the analysis presented here based on observatory data.

\section{Conclusions}

Based on 10 years of high-resolution CHAMP magnetic field data we have investigated the statistical properties of the lunar influence on the equatorial electrojet. This is the first study addressing the various aspects of lunar tides on global scale. From the results obtained a much clearer picture of the EEJ modulation emerges. Here we are listing some of the important findings:

1. The lunar tides cause an enhancement of the EEJ intensity at times when the moon is overhead or at the antipode. Tidal amplitudes are largest around December solstice and smallest around June solstice.

2. The tidal phase lags behind the moon phase. The delay is largest during December amounting to about $4 \mathrm{~h}$ of moon phase compared to other times of the year when it is $1-2 \mathrm{~h}$ of MP.

3. The lunar effect on the EEJ does not vary much with longitude. For that reason studies of the lunar tide can be performed by any observatory under the magnetic equator.

4. Solar activity is influencing the amplitude of the tidal variation, but the relative modulation of the EEJ intensity does not change significantly with solar flux level. During times of low solar activity lunar tidal features appear much clearer.

5. Our results suggest that gravitational forcing of the lower atmosphere by moon and sun causes an upward propagating semi-diurnal tidal wave which is driving a current at dip latitudes that adds to the EEJ. There is clear evidence that the lunar tide is not modulating the regular EEJ intensity.

6. The lunar influence on the EEJ is strictly different from the effect of non-migrating solar tides such as DE3. The effect of DE3 is at least double as strong on the EEJ intensity and the seasonal/longitudinal variations show a very different distribution.

7. We suggest that sudden stratospheric warming events are responsible for the enhancement of the lunar tidal amplitudes around December solstice. During SSW events the conditions for upward propagation of the tidal wave are probably improved.

8. Lunitidal phase delays show the same seasonal variation pattern in surface air pressure measurements as in the EEJ, i.e. larger delays in the months around December than during the rest of the year. But delays in the ionosphere are by about $2 \mathrm{~h}$ longer in moon phase.

For a validation of the suggestions made here lunar tidal signals in winds and temperature at the mesosphere, lower thermosphere region should be investigated. 
Acknowledgements. The CHAMP mission was sponsored by the Space Agency of the German Aerospace Center (DLR) through funds of the Federal Ministry of Economics and Technology, following a decision of the German Federal Parliament (grant code 50EE0944).

Topical Editor K. Kauristie thanks S. England and another anonymous referee for their help in evaluating this paper.

\section{References}

Alken, P. and Maus, S.: Spatio-temporal characterization of the equatorial electrojet from CHAMP, Ørsted, and SACC satellite measurements, J. Geophys. Res., 112, A09305, doi:10.1029/2007JA012524, 2007

Bartels, J.: Aufschlüsse über die Ionosphäre aus der Analyse sonnen- und mond-tägiger erdmagnetischer Schwankungen, Z . Geophys., 12, 368-378, 1936.

Bartels, J. and Johnston, H. F.: Geomagnetic tides in horizontal intensity at Huancayo, Part I, terrestrial magnetism and atmospheric electricity, J. Geophys. Res., 45, 269-308, 1940.

Bartels, J. and Kertz, W.: Gezeitenartige Schwingungen der Atmosphäre, in: Landoldt-Börnstein, Astronomie und Geophysik, edited by: Bartel, J. and Ten Bruggencate, P., pp. 674-685, Springer, Berlin-Göttingen-Heidelberg, 1952.

Chapman, S. and Lindzen, R.: Atmospheric Tides, pp. 66-105, Reidel, Dordrecht, Holland, 1970.

Chau, J. L., Fejer, B. G., and Goncharenko, L. P.: Quiet variability of equatorial E-B drifts during a sudden stratospheric warming event, Geophys. Res. Lett., 36, L05101, doi:10.1029/2008GL036785, 2009.

England, S. L., Maus. S., Immel, T. L., and Mende, S. B.: Longitude variation of the E-region electric fields caused by atmospheric tides, Geophys. Res. Lett., 33, L21105, doi:10.1029/2006GL027465, 2006.

Fejer, B. G., Olson, M. E., Chau, J. L., Stolle, C., Lühr, H., Goncharenko, L. P., Yumoto, K., and Nagatsuma, T.: Lunardependent equatorial ionospheric electrodynamic effects during sudden stratospheric warmings, J. Geophys. Res., 115, A00G03, doi:10.1029/2010JA015273, 2010.

Hagan, M. E. and Forbes, J. M.: Migrating and nonmigrating diurnal tides in the middle and upper atmosphere excited by tropospheric latent heat release, J. Geophys. Res., 107, 4754, doi:10.1029/2001JD001236, 2002.

Jadhav, G., Rajaram, M., and Rajaram, R.: A detailed study of equatorial electrojet phenomenon using Ørsted satellite observations, J. Geophys. Res., 107, 1175, doi:10.1029/2001JA000183, 2002.
Lühr, H., Maus, S., and Rother, M.: The noon-time equatorial electrojet, its spatial features as determined by the CHAMP satellite, J. Geophys. Res., 109, A01306, doi:10.1029/2002JA009656, 2004.

Lühr, H., Rother, M., Häusler, K., Alken, P., and Maus, S.: The influence of nonmigrating tides on the longitudinal variation of the equatorial electrojet, J. Geophys. Res., 113, A08313, doi:10.1029/2008JA013064, 2008.

Lühr, H., Rother, M., Häusler, K., Fejer, B., and Alken, P.: Direct comparison of non-migrating tidal signatures in the electrojet, vertical plasma drift and equatorial ionization anomaly, J. Atmos. Solar-Terr. Phys., 75-76, 31-43, doi:10.1016/j.jastp.2011.07.009, 2011.

Manoj, C., Maus, S., Lühr, H., and Alken, P.: Penetration characteristics of the interplanetary electric field to the daytime equatorial ionosphere, J. Geophys. Res., 113, A12310, doi:10.1029/2008JA013381, 2008.

Maus, S., Rother, M., Stolle, C., Mai, W., Choi, S.-C., Lühr, H., Cooke, D., and Roth, C.: Third generation of the Potsdam Magnetic Model of the Earth (POMME), Geochem. Geophys. Geosyst., 7, Q07008, doi:10.1029/2006GC001269, 2006.

Maus, S., Alken, P., and Lühr, H.: Electric fields and zonal winds in the equatorial ionosphere inferred from CHAMP satellite magnetic measurements, Geophys. Res. Lett., 34, L23102, doi:10.1029/2007GL030859, 2007.

Oberheide, J., Forbes, J. M., Häusler, K., Wu, Q., and Bruinsma, S. L.: Tropospheric tides from 80 to $400 \mathrm{~km}$ : Propagation, interannual variability, and solar cycle effects, J. Geophys. Res., 114, D00I05, doi:10.1029/2009JD012388, 2009.

Onwumechili, C. A.: Lunar effect on the diurnal variation of the geomagnetic horizontal field near the magnetic equator, J. Atmos. Terr. Phys., 25, 55-70, 1963.

Onwumechili, C. A.: The Equatorial Electrojet, pp. 254-270, Gordon and Breach, 1997.

Park, J., Lühr, H., Kunze, M., Fejer, B. G., and Min, K. W.: Effect of sudden stratospheric warming on lunar tidal modulation of the equatorial electrojet, J. Geophys. Res., 117, A03306, doi:10.1029/2011JA017351, 2012.

Rastogi, R. G. and Trivedi, N. B.: Luni-solar tides in $\mathrm{H}$ at stations within the equatorial electrojet, Plant. Space Sci., 18, 367-377, 1970.

Reigber, C., Lühr, H., and Schwintzer, P.: CHAMP mission status, Adv. Space Res., 30, 129-134, 2002.

Robinson, R. M. and Vondrak, R. R.: Measurements of E region ionization and conductivity produced by solar illumination at high latitudes, J. Geophys. Res., 89, 3951-3958, 1984.

Schlegel, K.: Auroral zone E-region conductivities during solar minimum derived from EISCAT data, Ann. Geophys., 6, 129$138,1988$. 